\title{
Descripción de un Nuevo Método para Clasificación de la Enfermedad Hipertensiva Inducida por el Embarazo
}

\author{
Dr. Alfonso Correa Uribe*
}

\begin{abstract}
O
Se revisaron las historias clínicas de las pacientes que presentaron Enfermedad Hipertensiva Inducida por el Embarazo (Pre-eclampsia - Eclampsia) en un período de 18 meses comprendido entre el 31 de diciembre de 1984 y el 30 de junio de 1985. En dicho período se encontró un total de 147 pacientes con diagnóstico de EHIE, de las cuales no se incluyeron en el estudio 40 pacientes, por no cumplir con los parámetros establecidos por nuestra Institución para el Diagnóstico de Preeclampsia - Eclampsia. Se realizó un estudio estadístico de los 107 casos restantes, de los cuales 12 correspondieron a Eclampsia.
\end{abstract}

Se realizó tambień un análisis discriminante de diferentes variables involucradas en la enfermedad para valorar la significancia que tiene cada una de estas con respecto a la severidad de la pre-eclampsia.

Se pretendió de esta forma buscar la importancia de cada una de estas variables

* Instructor Asociado de Gineco-Obstetricia Colcgio Mayor de Nuestra Señora del

Rosario - Hospital San José de Bogotá por medio de una combinación lineal. La función discriminante para cada variable se obtuvo mediante método directo y método stepwise. Por medio del método directo no se obtuvieron datos de importancia. Con el método de Stepwise se encontró que las variables más significativas fueron la edad de la paciente, número de gestaciones previas y edad gestacional al desembarazar a la paciente. No se encontró significancia estadística en los niveles séricos de Acido Urico ni de Plaquetas. Esto simplemente indica que hay variables que tienen una gran importancia clínica mas no tienen una importancia estadística. Se hallaron dos funciones canónicas por medio de estas variables descritas anteriormente. Con estas funciones se logra discriminar en forma precisa los diferentes niveles de severidad de la enfermedad. Estas dos funciones discriminantes se pueden apreciar mejor por medio de un mapa territorial, mediante el cual se puede ubicar si la paciente en cuestión padece determinada severidad de pre-eclampsia.

\section{INTRODUCCION}

La Enfermedad Hipertensiva Inducida por el Embarazo es una de las patologías 
obstétricas más frecuentes en nuestro medio. Igualmente es uno de los temas más controvertidos en la actualidad y existen de élla múltiples formas de clasificación, diferentes estudios diagnósticos paraclínicos y variados protocolos de manejo. Todos los autores que hemos consultado le dan valores distintos a los exámenes paraclínicos y a los hallazgos clínicos, sin que exista un acuerdo entre ellos, de cuál de éstos es el más significativo. Weinstein y cols. describieron dentro de la EHIE un síndrome caracterizado por trombocitopenia hemolítica y enzimas hepáticas alteradas, el llamado Síndrome HELLP y es por esto que este grupo de investigadores le dan gran importancia a los niveles de plaquetas y a los demás parámetros para-clínicos que nos indican las alteraciones de dicho síndrome. Los Doctores Burrow y Ferris en su libro de "Complicaciones Médicas y Embarazo" proponen una clasificación de la enfermedad basándose en parámetros paraclínicos. Proponen que niveles de Acido Urico menores de 4,5 mg\%corresponden a Pre-eclampsia leve, niveles entre 4,5 y 5,5 mg\% corresponden a Pre-eclampsia moderada y los niveles superiores a 5,5 a Pre-eclampsia severas. Motivados por estos autores, los médicos del Departamento de Gineco-Obstetricia del Hospital de San José, hemos venido solicitando rutinariamente dentro de los exámenes para-clínicos de ingreso de las pacientes con EHIE, niveles séricos de Acido Urico y Plaquetas.

Es uno de los propósitos de este trabàjo determinar cuál es la importancia que tienen estos exámenes y otras variables para la clasificación de la enfermedad.

\section{MATERIAL Y METODOS}

Se revisaron las historias clínicas de las pacientes que egresaron con diagnóstico de Pre-eclampsia - Eclampsia, en un período de 18 meses, comprendido entre el 31 de diciembre de 1984 y el 30 de junio de 1986. Se encontraron un total de 147 pacientes con diagnóstico de egreso de Enfermedad Hipertensiva Inducida por el Embarazo (EHIE). Para el diagnóstico de EHIE en nuestra Institución, se requiere que la paciente cumpla con las siguientes condiciones:

1. Hipertensión Arterial: TA $>$ de 140/ 90 , o aumento de la cifra sistólica de más de $30 \mathrm{mmHg}$ o de la diastólica de más de $15 \mathrm{mmHg}$, y que este aumento en la tensión sea sostenido, o sea que se mantenga después de 6 horas de reposo.

2. Proteinuria: más de $30 \mathrm{mg} \%$ en un Parcial de Orina aislado.

3. Edemas: matutinos o que prevelezcan después de 12 horas de reposo.

De las 147 pacientes recolectadas, 40 no se incluyeron en el estudio por no cumplir con los parámetros anteriormente descritos.

Los 107 casos restantes correspondían a 12 casos de Eclampsia (11.21\%), $36 \mathrm{ca}-$ sos de Pre-eclampsia Severa (33,64\%), 34 casos de Pre-eclampsia Moderada $(31,78 \%)$ y 25 casos de Pre-eclampsia Leve $(23,36 \%)$. Esto nos demuestra que la mayoría de las pacientes presentaban grados moderados y severos de la enfermedad.

Para el análisis estadístico se tomaron las siguientes variables:

1. Edad de la Paciente VI

2. Número de Gestaciones previas $\vee 2$

3. Número de Partos previos V3

4. Número de Abortos previos V4

5. Edad Gestacional de aparición de la enfermedad V5. 
6. Edad Gestacional al desembarazar a la paciente V6.

7. Niveles séricos de Acido Urico V7.

8. Cuantificación de Plaquetas V8.

Para la determinación de estas dos últimas variables se utilizaron los métodos realizados comúnmente por el Laboratorio Clínico del Hospital.

En la realización del estudio estadístico contamos con la colaboración de un Ingeniero Industrial. Este estudio fue desarrollado en el Departamento de Sistemas de la Universidad de los A.ndes, mediante el paquete estadístico SPSS (Statistical Package for Social Sciences) en un computador Burroughs B 6800 .

EI análisis estadístico de los datos se realizó de dos formas:

a) Análisis Estadístico descriptivo.

b) Análisis Discriminante de las variables.

\section{Análisis Estadístico Descriptivo}

Se realizó un análisis estadístico descriptivo en forma usual. También se hizo un estudio con tablas de contingencia para medir la dependencia de cada una de las variables con respecto a la severidad. Para esto se utilizó la prueba de Chi cuadrádo y una significancia de 0,99.

\section{Análisis Discriminante}

El objetivo del análisis discriminante es pesar y hacer una combinación lineal de las variables más significativas con las cuales se logra la mayor diferenciación entre los grupos de clasificación de la severidad de la enfermedad. Se pueden tener una o más combinaciones lineales de las variables discriminantes significativas. El máximo número de funciones discriminantes es uno menos que el número de gruposo igual al número de variables discriminantes, si hay más grupos que variables. Para este estudio no se tomó en consideración la Eclampsia, ya que se trata de una entidad de fácil reconocimiento clínico. De tal manera que se tienen en este caso tres grupos (leve, moderada, severa) y ochovariables, por lo que el número de funciones discriminantes es igual a dos. Dichas funciones deben estar conformadas por aquellas variables que maximizan la separación entre los grupos. Una vez se tengan las funciones discriminantes, se pueden lograr los dos principales objetivos del análisis discriminante: analizar y clasificar.

El análisis consiste en utilizar las diferentes estadísticas con las funciones para interpretar los datos. En cuanto a los coeficientes de las funciones discriminantes, pueden ser utilizados para identificar aquellas variables que más contribuyen a la diferenciación entre los grupos de clasificación de la enfermedad.

Para obtener la función discriminante se pueden utilizar diferentes métodos. El METODO DIRECTO, el cual consiste en incluir todas las variables discriminantes en la función. Este método no indica qué variables son realmente significativas para la clasificación de la enfermedad. El METODO STEPWISE consiste en ir incluyendo en la función discriminante aquellas variables que son significativas. Las funciones discriminantes se expresan de la siguiente forma:

$$
\begin{aligned}
& D=d Z+d Z+\ldots \ldots \ldots d z \\
& \text { i i1 i i } 22 \text { ipp }
\end{aligned}
$$

donde Di es el resultado de la función discriminante $\mathrm{i}, \mathrm{dij}=$ coeficiente de peso y $\mathrm{Zj}=$ valores estandarizados de las 
j variables discriminantes usadas en el análisis.

El criterio de selección se basa en la estadística $\mathrm{F}$ de cada una de estas variables. Si la $F$ de la variable es menor que 1 , dicha variable no se incluye en la función. Se toman en cuenta solamente las variables que tienen la estadística $\mathrm{F}$ mayor que 1. En cada paso sólo se incluye una sola variable, o se remueve si es el caso.

La representación espacial de las funciones discriminantes se conoce como Mapa Territorial. En este caso, esta representación es un plano de 2 dimensiones, puesto que como se verá en los resultados, se obtuvieron dos diferentes funciones discriminantes. En el mapa territorial se puede apreciar la dispersión de los grupos que se desean diferenciar, según el rango que ocupan los grupos dentro del rango determinado por las funciones discriminantes. Este rango en este mapa es Fie $(-4,+4)$; F2e $(-4,+4)$;

\section{RESULTADOS}

\section{Análisis Descriptivo}

Durante los 18 meses estudiados se atendieron en el hospital 8248 partos. En dicho período se encontraron 107 pacientes con Enfermedad Hipertensiva Inducida por el Embarazo, lo que da una incidencia de la enfermedad del $1.29 \%$.

Se interpretaron como Pre-eclámpticas 147 pacientes en total, 40 de las cuales no cumplían los requisitos indispensables para diagnosticar la enfermedad, lo cual equivale a un error de diagnóstico del 27 , $21 \%$, cifra ésta que está de acuerdo con lo encontrado por Chesley en la revisión hecha por él en 1984.
De las 107 pacientes con EHIE, 12 correspondieron a pacientes Eclámpticas $(11.21 \%)$.

La edad promedio de las pacientes eclámpticas fue de 23.6 años, con un rango de 17 a 40 años. Dos de estas pacientes se encontraban con tensiones arteriales normales (120/80), y una tenía cifras diastólicas de $100 \mathrm{mmHg}$. Las restantes $9 \mathrm{pa}$ cientes tenían cifras diastólicas mayores de $110 \mathrm{mmHg}$.

El tratamiento en las 12 pacientes eclámpticas consistió en disminuir la tensión arterial mediante bolos de apresolina, cuando la cifra diastólica era mayor de $110 \mathrm{mmHg}$, controlar las convulsiones, y una vez se estabilizó el cuadro clínico se desembarazaron las pacientes. De las 12 pacientes, 6 recibieron Sulfato de Magnesio al 20\%, 4 a 6 gms, iniciales únicamente. Ninguna paciente recibió Sulfato de Magnesio en goteo de mantenimiento. No se utilizó Sulfato de Magnesio de mantenimiento, ya que en nuestro hospital no contamos con la posibilidad de hacer Magnesemias a las pacientes, ni con las suficientes bombas de infusión para garantizar una dosis constante. Sabemos que las dosis terapéuticas son muy cercanas a las dosis tóxicas y por este motivo preferimos utilizar otro tipo de anti-convulsivante en el control de estas pacientes. Siete de las pacientes recibieron Epamin endovenoso como tratamiento para su estado convulsivo, siguiéndose esta droga de mantenimiento en el puerperio inmediato. 5 pacientes recibieron Valium endovenoso en el momento de la crisis convulsiva únicamente, y 1 paciente recibió Fenobarbital 400 mgs. parenterales. No se encontró ninguna diferencia mayor en cuanto al éxito de los diferentes tratamientos anti-convulsivantes, ya que se lograron controlar los episodios convulsivos en la mayoría de los casos, excepto en una paciente con 
Síndrome HELLP, quien presentó crisis convul sivas a repetición y una paciente que presentó un Status Epiléptico. Creemos que para el manejo de las convulsiones es mejor utilizar drogas que no interfieran con el estado de conciencia de la madre, ya que si esto sucediera, sería muy difícil establecer si dicho trastorno se debe a la droga o a complicaciones propias de la Eclampsia (A.C.V. e.t.c.). Ninguna paciente con pre-eclampsia leve o moderada recibió Valium o Fenobarbital parenteral o Vía Oral.

Las complicaciones de las pacientes Eclámpticas fueron muerte materna en 1 caso, y Status Epiléptico en 1 caso que obligó a la administración de penthotal. (Tabla No. 1).

De las 12 pacientes Eclámpticas, 3 correspondieron a Síndrome HELLP. Se encontró dicho síndrome en 2 pacientes con
Preeclampsia severa. De las 5 pacientes con síndrome HELLP 1 murió luego de convulsionar en cuatro oportunidades. Al parecer la causa de muerte fue una hemorragia cerebral, aunque no fue confirmada por autopsia. Otra paciente con Síndrome HELLP presentó Insuficiencia Renal Aguda, Necrosis Cortical e Insuficiencia Renal Crónica posterior. Actualmente se encuentra en programa de diálisis crónica en el Hospital. Esta paciente se había clasificado como Pre-eclampsia Severa, basados en la sintomatología y en las cifras tensionales al ingreso de la paciente. (Tabla No. 2).

En cuanto a los resultados del análisis de las tablas de contingencia, se encontró lo siguiente:

De las 8 variables analizadas se encontró que no existía relación entre la variable $y$ la severidad de la enfermedad en 4 de

TABLA No. 1

\section{PACIENTES ECLAMPTICAS}

\begin{tabular}{|c|c|c|c|c|c|c|c|c|}
\hline$E D A D$ & $T . A$. & AFFES = & SLILFAIO & GOTEO SULF. & WAL IEAY & EFAMIN & FET & COMPLILAC \\
\hline 28 & $22 / 14$ & sI & SI & NO & 51 & si & no & no \\
\hline 20 & $13 / 10$ & NO & $S I$ & NO & $\mathrm{Si}$ & SI & no & NO \\
\hline 22 & $16 / 12$ & $8 \mathrm{I}$ & $\mathrm{NO}$ & no & NO & Mo & $S I$ & MUEFTE \\
\hline 30 & $22 / 14$ & SI & NO & NO & no & no & NO & NO \\
\hline 27 & $16 / 12$ & $S I$ & 140 & NO & No & $S I$ & NO & 140 \\
\hline 18 & $15 / 11$ & SI & NO & NO & No & NO & NO & No \\
\hline 17 & $20 / 13$ & $\mathrm{SI}$ & No & No & $S I$ & SI & no & no \\
\hline 20 & $19 / 12$ & SI & $S I$ & No & $B I$ & Si & NO & $\mathrm{No}$ \\
\hline 40 & $12 / 8$ & NO & SI & NO & 51 & $S I$ & NO & SIAT.EF. \\
\hline 24 & $12 / 8$ & No & No & NO & 50 & No & $\mathrm{Si}$ & no \\
\hline 20 & $15 / 11$ & $\subseteq i$ & NO & No & $\mathrm{NO}$ & SI & NO & NO \\
\hline 18 & $15 / 11$ & si & SI & NO & $\mathrm{SI}$ & no & No & $\mathrm{Nin}$ \\
\hline
\end{tabular}


TABLA No. 2

SINDROME HELLP

\begin{tabular}{|c|c|c|c|c|c|c|}
\hline EDAD & GESTALIONES & EDADX & GEST. & FLAGUETAS & SEVERIIAD & COMFLICACIOUES \\
\hline 20 & G1FO & 31 & sem. & 98.000 & Eci ampsia & Ninguna \\
\hline 22 & $B 1 F 0$ & 32 & 2 sem. & 54.000 & Ecl ampsia & Huerte Hat. \\
\hline $2 \%$ & GSF 1 & 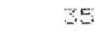 & $=\operatorname{sen}$. & 91.000 & F.E. Sever a & I. F.A. \\
\hline 28 & $54 \mathrm{FB}$ & 32 & sem. & 52.000 & Eclampsia & Ningina \\
\hline 32 & $\mathrm{G} 4 \mathrm{P2}$ & 32 & 2 sem. & 66.000 & F. E. Severa & Nirguna \\
\hline
\end{tabular}

ellas: V1, V2, V3, V8. La edad gestacional de aparición se correlacionó con la severidad de la enfermedad con una significancia de 0,99 ( $p<0,01)$, lo cual quiere decir que entre menor sea la edad gestacional a la que aparece la enfermedad, mayor probabilidad hay de que sea Severa o Eclampsia. 48,6\% de las pacientes con Preeclampsia severa se presentaron antes de la semana 34 de gestación, y $58.3 \%$ de las pacientes con Eclampsia se presentaron antes de la semana 34, mientras que no hubo ningún caso de Pre-eclampsia leve en dichas semanas de gestación. Por otra parte, $84 \%$ de las pacientes con Pre-eclampsia leve consultarún iuego de la semana 37, y sólo se encontraron $35.1 \%$ y $25 \%$ de pacientes con Pre-eclampsia severa y Eclampsia respectivamente en dichas semanas de gestación (Figura No. 8). Otra de las variables que demostró tener relación con respecto a la severidad de la enfermedad fue la Edad Gestacional a la cual se desembarazaron las pacientes. Este resultado en cierta forma es obvio, ya que el manejo de la EHIE en nuestra institución se basa en desembarazar precozmente a la paciente. Antes de la semana 34 se desembarazaron 38,8\% de las pacientes con Pre-eclampsia severa y $50 \%$ de pacientes con Emclapsia. Esto demuestra que sólo el 18,8\% de Pre-eclampsias severas ( 7 casos) y el 8,3\% de Eclamp- sias ( 1 caso), que aparecieron antes de las 34 semanas se manejaron en forma conservadora.

Se encontraron 2 variables que se correlacionaron con la severidad de la enfermedad pero no con una significancia del 0,99 . La edad de la paciente se correlacionó con la severidad de la enfermedad pero con una significancia de 0,95 ( $p<0,05)$, lo cual no es estadísticamente significativo. De igual forma el Acido Urico se correlacionó con la severidad de la enfermedad con una significancia de 0,95. Esto quiere decir que existe un $95 \%$ de probabilidad que entre mayor sea el nivel sérico de A. Urico, mayor es la severidad de la enfermedad (Figura No. 10).

\section{Análisis Discriminante}

Según el método directo no se obtuvieron resultados de importancia.

Sin embargo, con el método Stepwise las variables discriminantes que resultaron más significativas fueron la edad de la paciente, el número de gestaciones previas y la edad gestacional al desembarazar.

De las dos posibles funciones discriminantes que se podían obtener (tres grupos 
- uno), se obtuvo ese máximo: dos funciones diferentes que utilizan las variables
V1, V2 y V6, cada una de ellas representada de la siguiente manera:

$$
\begin{array}{ll}
\mathrm{FI}=0.816(\mathrm{~V} 2)-0.309(\mathrm{~V} 1)+0.856(\mathrm{~V} 6) & \begin{array}{l}
\mathrm{V} 1=\text { Edad pacien. } \\
\mathrm{V} 2=\text { No. de Gestac. }
\end{array} \\
\mathrm{F} 2=0.184(\mathrm{~V} 2)-0.836(\mathrm{~V} 1)+0.221(\mathrm{~V} 6) & \mathrm{V} 6=\mathrm{EG} \text { Desembar. }
\end{array}
$$

F1 es una función discriminante donde se le da mayor relevancia a las gestaciones previas y a la edad gestacional al desembarazar. F2, por otra parte, es una función donde se le da relevancia a la edad de la paciente.

Utilizadas las dos en conjunto, se logra diferenciar los grupos en forma adecuada.

Para un mejor entendimiento de estas dos funciones, se puede utilizar el mapa territorial donde la abscisa es una función y la coordenada es la otra función. Véase Figura No. 12.

El mapa territorial refleja el al to grado de discriminación entre los grupos, dado por las variables discriminantes, que actúan como variables dependientes en las funciones discriminantes. Los grupos no presentan entrecruzamiento de rangos, cada uno ocupa un espacio definido en el mapa. Este espacio se puede apreciar en la Figura No. 12.

$$
\begin{array}{rr}
\text { Rangos: Para F1: Grupo } 1 & (-0,9 ;+4) \\
\text { Grupo } 2 & (-0,8 ;+4) \\
\text { Grupo } 3 & (-1 ;-0,1 \\
\text { Para F2: Grupo 1 } & (-0,1 ;+4 \\
\text { Grupo 2 } & (-0,2 ;-4) \\
\text { Grupo 3 } & (-4 ;+4)
\end{array}
$$

El mapa territorial corrobora la discriminación entre los 3 grupos que se había concluido del análisis discriminante (de las estadísticas obtenidas), dado por la relación lineal entre la función y las variables discriminantes.

\section{COMENTARIOS}

El análisis estadístico puede ser de gran utilidad práctica para la clasificación posterior de las pacientes con EHIE ya que algunas de ellas se presentaron con cuadros ambivalentes, donde era difícil determinar si se trataba de uno u otro grado de severidad. Es en esta paciente donde un método objetivo basado en una fórmula estadística nos puede orientar. Por ejemplo, aquella paciente joven, con una o dos gestaciones previas, quien refería una cefalea moderada y cuya tensión arterial en el momento de la consulta se encontró en 120/ 80 pero desconocíamos los valores previos; de igual manera la paciente gran multípara, con cefalea leve (subjetivo) pero en quien encontramos cifras tensionales de 170/110. Estas son pacientes que si nos guiáramos por sólo un parámetro, la clasificaríamos en diferentes grados de severidad dependiendo de la variable que empleemos. Sin embargo, si en estas pacientes utilizamos la función discriminante, tomando el valor del centroide para el grupo en cuestión en el mapa territorial, nos da un dato más para clasificar a esta paciente en un grado determinado de severidad, el cual unido a todos los datos clínicos y paraclínicos, nos indica en qué grado de severidad se encontraba realmente la enfermedad.

En este trabajo se hizo el análisis en forma retrospectiva, pero se debe realizar un nuevo estudio en el cual no se incluya la variable de edad gestacional al desembarazar, para tratar de determinar si exis- 
ten variables discriminantes que se puedan incluir en funciones discriminantes que sean utilizables en forma prospectiva y de esta forma clasificar a la paciente por medio de una fórmula estadística, de tal manera que se pueda prever cuándo es la edad gestacional adecuada para desembarazarla. Esto implicaría tomar a la edad gestacional de desembarazo como un grupo más y no como una variable. Esto será tema de un futuro análisis cuidadoso, donde se involucren más datos.

Quiero expresar mis agradecimientos al Sr. Diego Correa Uribe, Ingeniero Indus- trial de la Universidad de los Andes, quien prestó su invaluable colaboración para el análisis estadístico de este trabajo, así como a la Sra. Elsa Victoria Correa Uribe, Ingeniero de Sistemas de la Universidad de los Andes, quien colaboró en la elaboración de las gráficas y tablas. Igualmente, quiero agradecer al Departamento de Estadística del Hospital de San José y a los Dres. Ivette Maldonado y Luis Clovis Torres, Gineco-obstetras de la Universidad del Rosario, quienes me ayudaron a la recolección de los datos. 

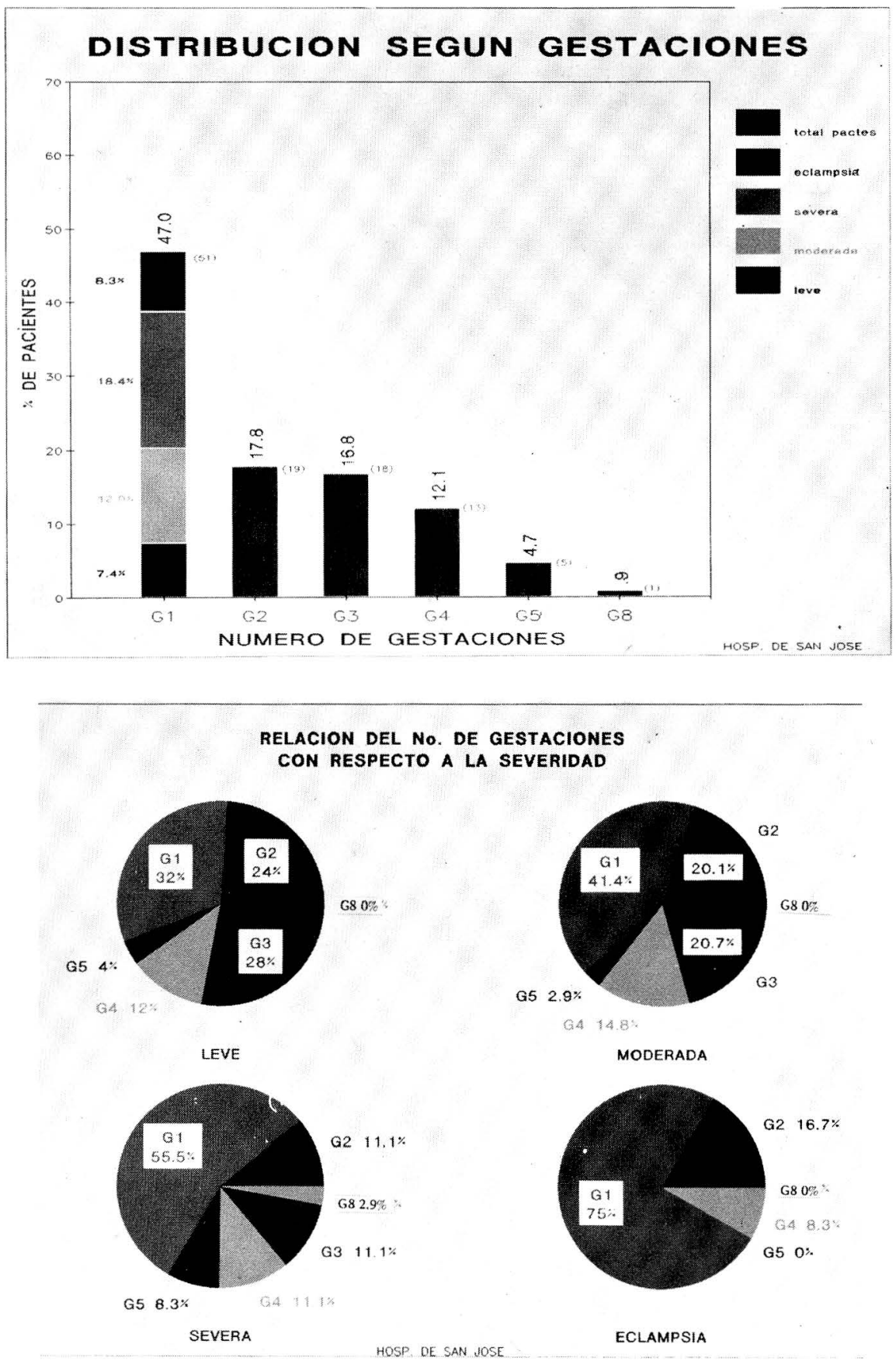
RELACION DEL. No. DE PARTOS CON RESPECTO A LA SEVERIDAD

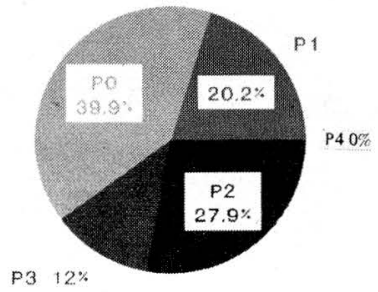

LEVE

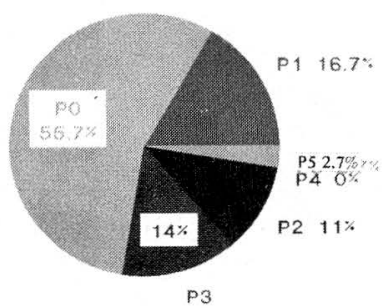

SEVERA

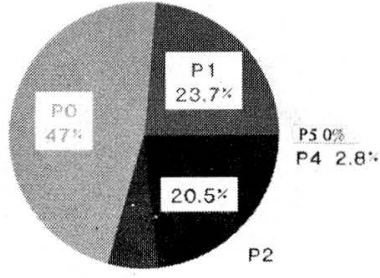

P:3 6\% MODERADA

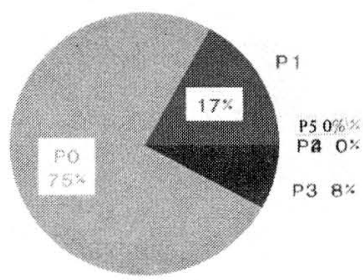

ECLAMIPSIA

\section{DISTRIBUCION SEGUN ABORTOS}

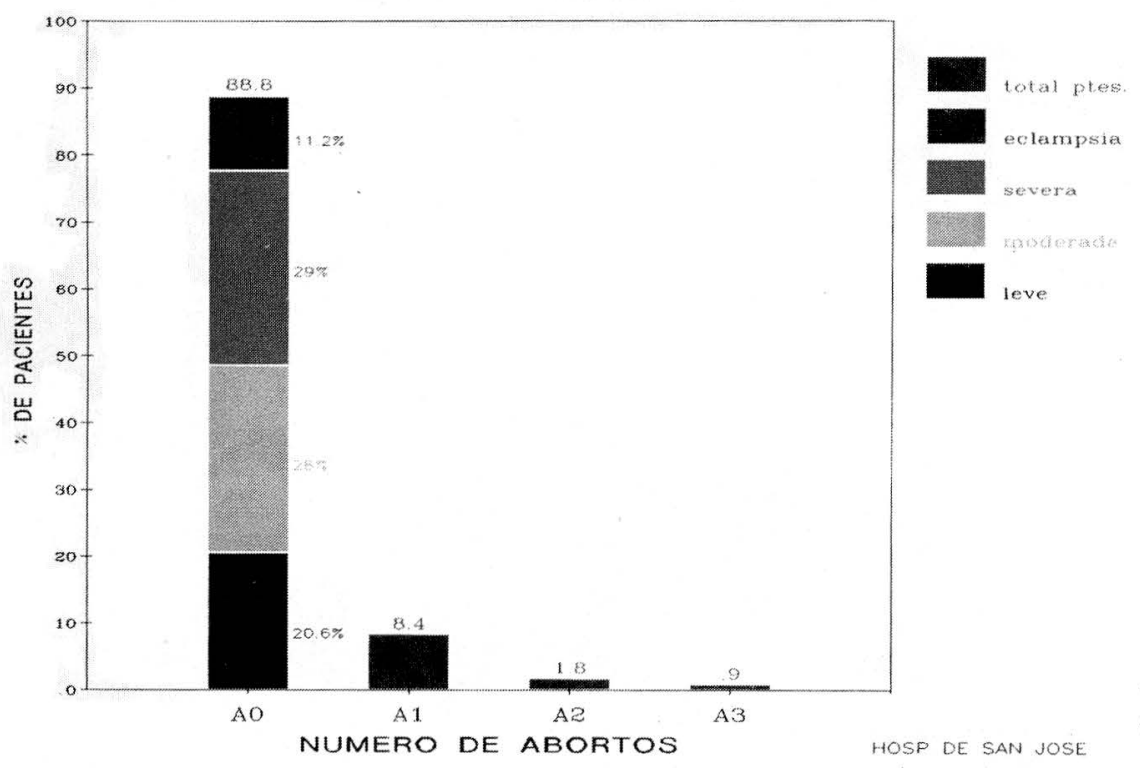


RELACION DE ABORTOS CON RESPECTO A LA SEVERIDAD
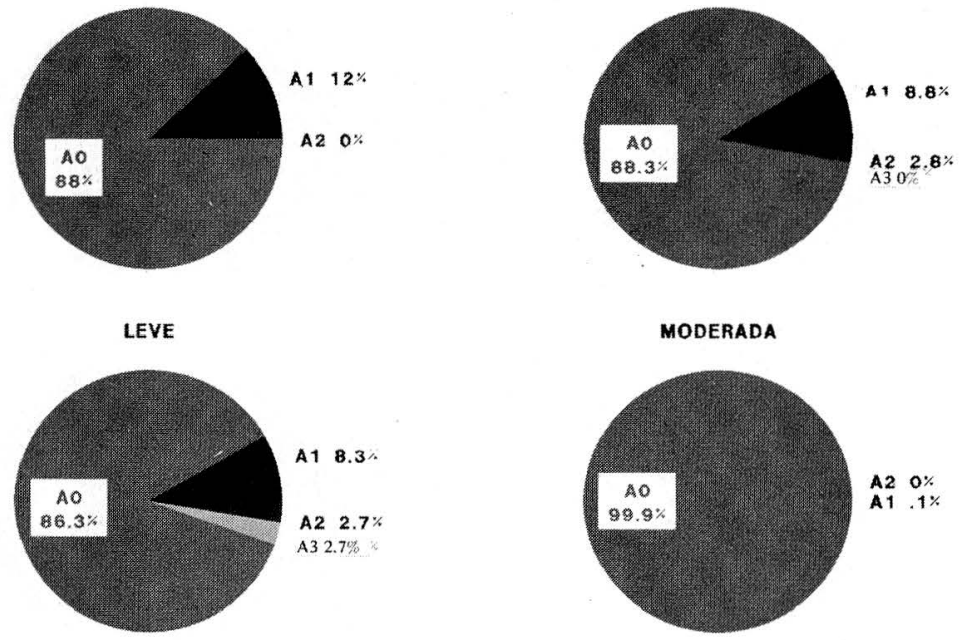

SEVERA

HOSP. DE SAN JOSE

ECLAMPSIA

Distribucion de la MUESTRA POR EDADES

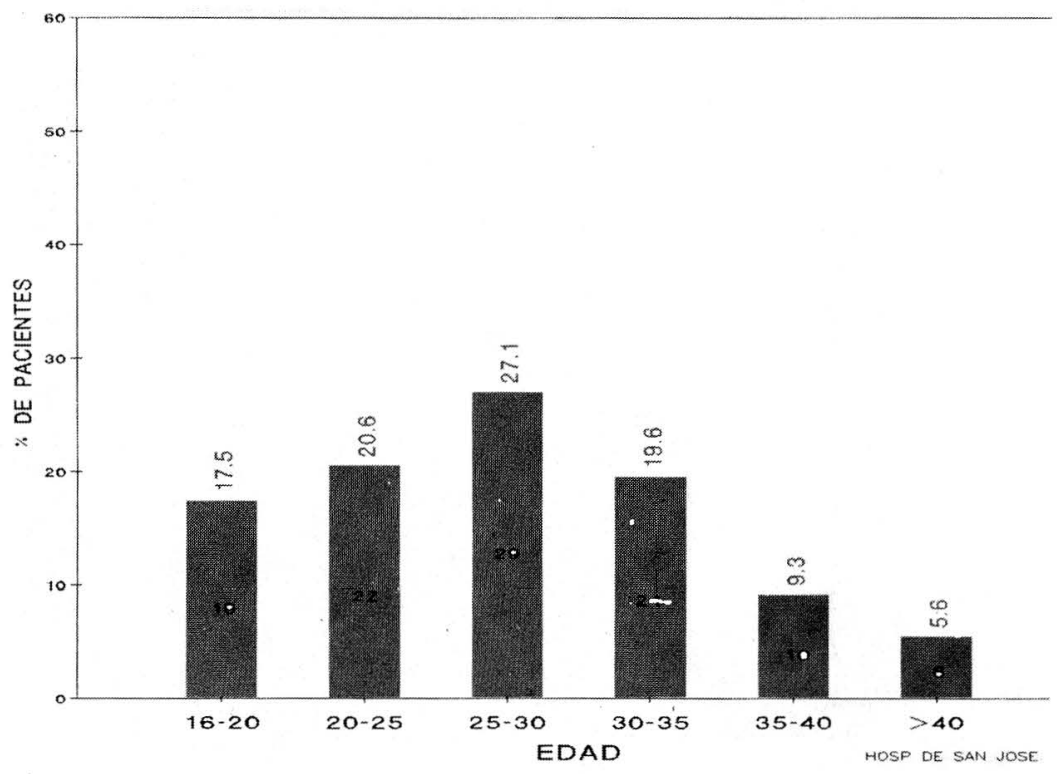


RELACION DE LA EDAD DE LA PACIENTE CON RESPECTO A LA SEVERIDAD

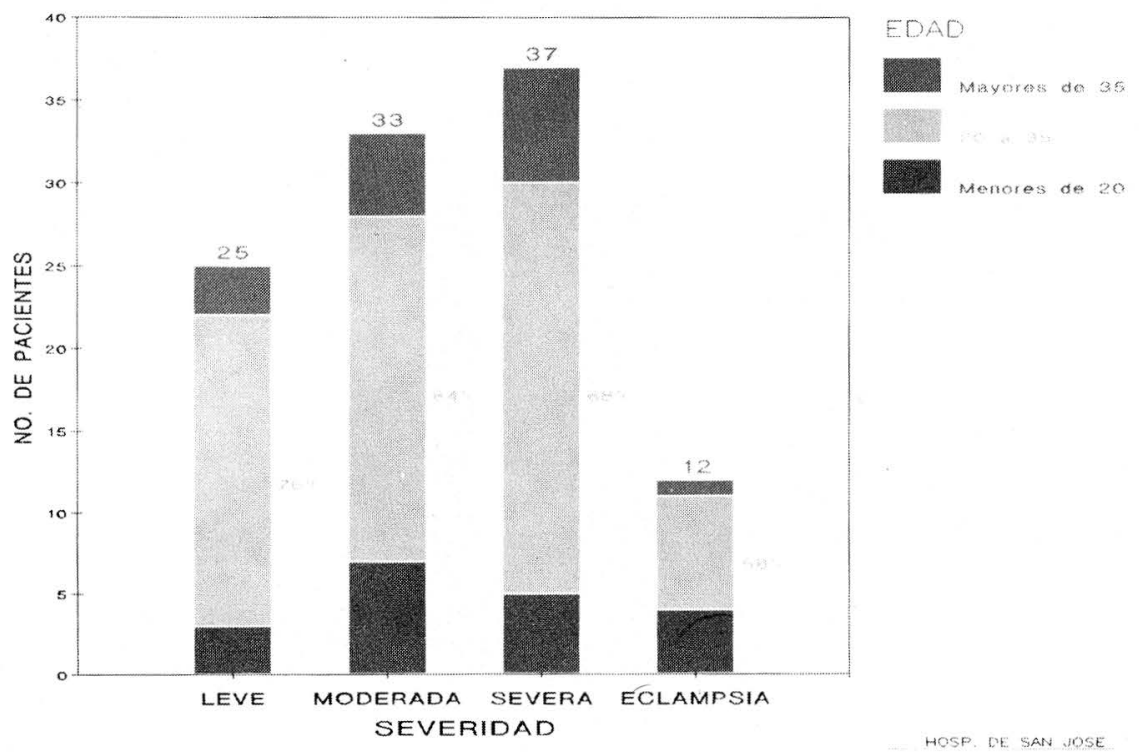

SEVERIDAD DE LA ENFERMEDAD CON RELACION EDAD GESTACIONAL DE APARICION

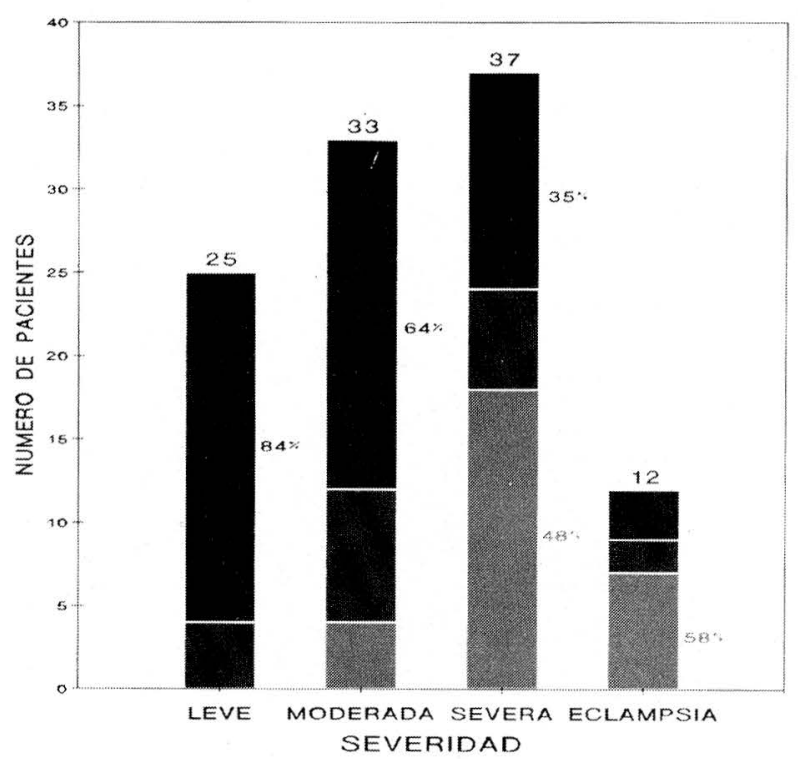

EDAD GESTACIONAI.

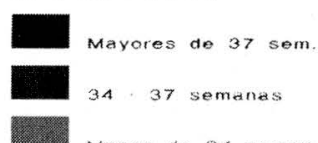




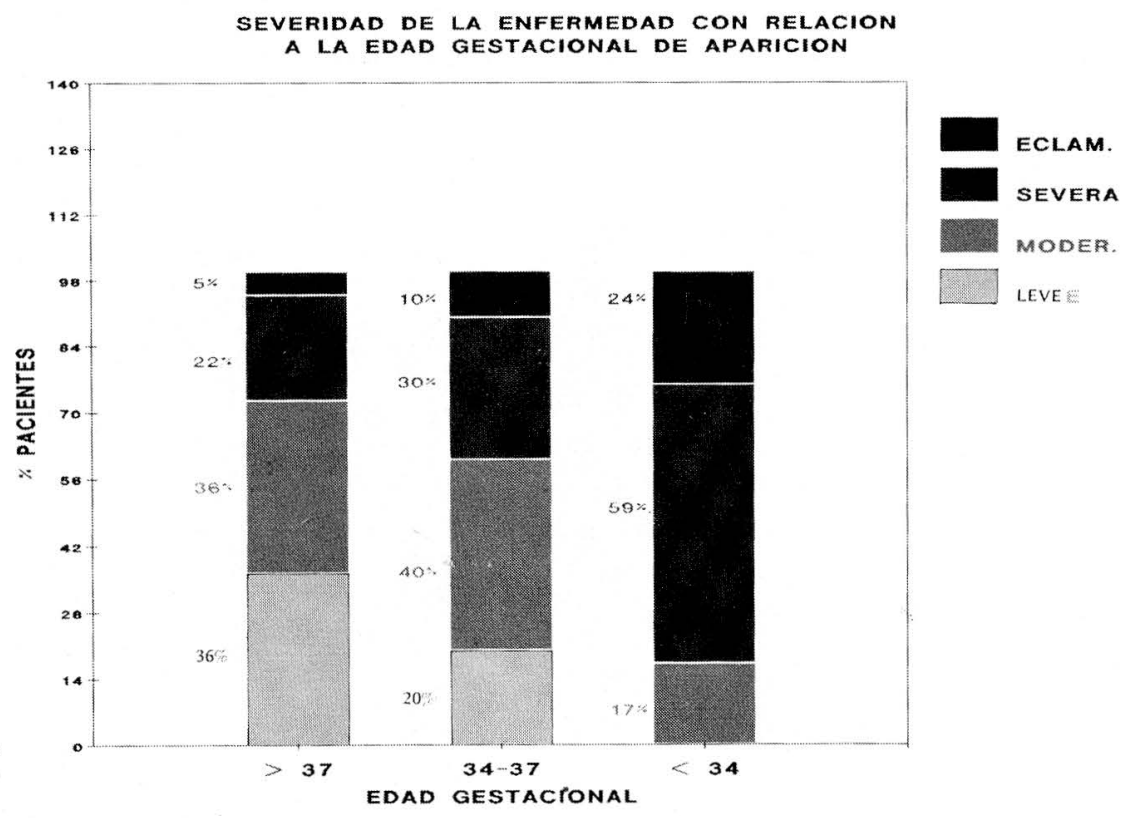

ACIDO URICO VS SEVERIDAD

\begin{tabular}{|c|c|c|c|c|}
\hline $\begin{array}{l}\text { severidad } \\
\mathrm{mg} \%\end{array}$ & LEVE & MODER. & SEVERA & ECLAMPSIA \\
\hline$<4$ & & & $3.3 \%$ & \\
\hline $4-6$ & $43.7 \%$ & $22.6 \%$ & $16.7 \%$ & $11.1 \%$ \\
\hline $6-8$ & $43.7 \%$ & $41.9 \%$ & $36.6 \%$ & $22.2 \%$ \\
\hline \multirow[t]{2}{*}{$>8$} & $12.5 \%$ & $35.5 \%$ & $43.3 \%$ & $66.7 \%$ \\
\hline & $100 \%$ & $100 \%$ & $100 \%$ & $100 \%$ \\
\hline
\end{tabular}


LEVE

TODEFADA

SEVEFIA

ECLAMFSIA

\begin{tabular}{|c|c|c|c|c|}
\hline$=250.00$ & $22 \cdot 2 \%$ & $17.2 \%$ & $6.2 \%$ & $16.6 \%$ \\
\hline $250-1=0$ & $\Rightarrow 1.1 \%$ & $69.2 \%$ & $59.5 \%$ & $41.7 \%$ \\
\hline $\pm 50-100$ & $11.1 \%$ & i1. $5 \%$ & $28.2 \%$ & $25.0 \%$ \\
\hline$<\mathrm{iol} \cdot 000$ & $5.5 \%$ & $0 \%$ & s. $2 \%$ & $10.6 \%$ \\
\hline
\end{tabular}

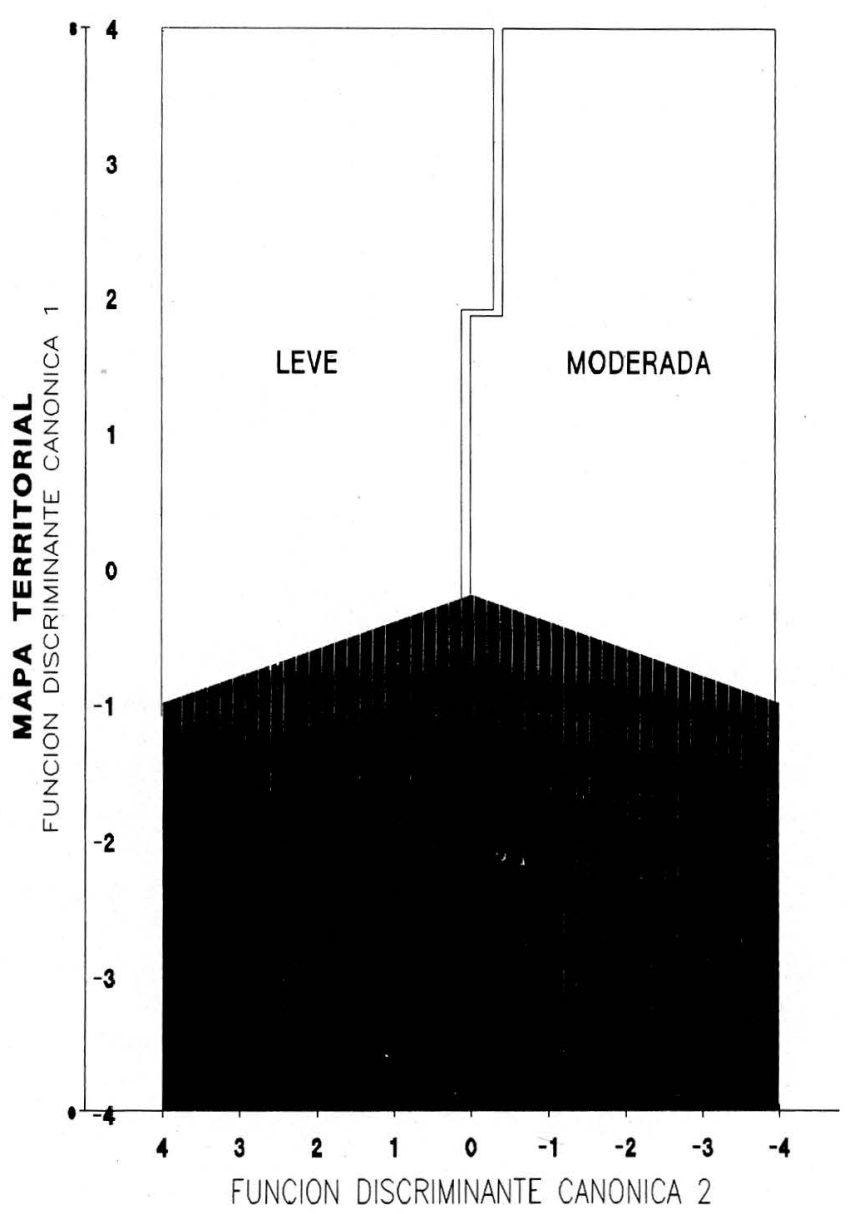

